\title{
Optimal Tuning of PID Controller Using Grey Wolf Optimizer Algorithm for Quadruped Robot
}

\author{
M. A. Şen, and M. Kalyoncu
}

\begin{abstract}
The research and development of quadruped robots is grown steadily in during the last two decades. Quadruped robots present major advantages when compared with tracked and wheeled robots, because they allow locomotion in terrains inaccessible. However, the design controller is a major problem in quadruped robots because of they have complex structure. This paper presents the optimization of two PID controllers for a quadruped robot to ensure single footstep control in a desired trajectory using a bio-inspired meta-heuristic soft computing method which is name the Grey Wolf Optimizer (GWO) algorithm. The main objective of this paper is the optimization of $K_{P}, K_{I}$ and $K_{D}$ gains with GWO algorithm in order to obtain more effective PID controllers for the quadruped robot leg. The importance to this work is that GWO is used first time as a diversity method for a quadruped robot to tune PID controller. Moreover, to investigate the performance of GWO, it is compared with widespread search algorithms. Firstly, the computer aided design (CAD) of the system are built using SolidWorks and exported to MATLAB/SimMechanics. After that, PID controllers are designed in MATLAB/Simulink and tuned gains using the newly introduced GWO technique. Also, to show the efficacy of GWO algorithm technique, the proposed technique has been compared by Genetic Algorithm (GA) and Particle Swarm Optimization (PSO) algorithm. The system is simulated in MATLAB and the simulation results are presented in graphical forms to investigate the controller's performance.
\end{abstract}

Index Terms- Quadruped Robot, PID controller, Optimization, Gait definition, Grey Wolf Optimizer, Genetic Algorithm, Particle Swarm Optimization, Trajectory Tracing.

\section{INTRODUCTION}

$\mathrm{T}$ RACEKED and wheeled robots can travel very fast only on fairly flat floors. However, they can move slower than legged robots on rougher terrains. Quadruped robots have the advantages of the strong obstacle capability, less energy consumption, high flexibility, good stability and locomotion on uneven and rough terrain, do little damage to the environment etc. So, quadruped robots are an important place in robotic and their popularity are increasing. Nevertheless, quadruped robots have more complicated structure and it is more difficult to control than tracked and wheeled robots.

M.A. ŞEN, is with Department of Mechanical Engineering University of Selçuk University, Konya, Turkey, (e-mail: marifsen@selcuk.edu.tr) (DD)

M. KALYONCU, is with Department of Mechanical Engineering University of Selçuk University, Konya, Turkey, (e-mail: mkalyoncu@ selcuk.edu.tr) iD

Manuscript received September 6, 2017; accepted January 11, 2018. DOI: $10.17694 /$ bajece.401992
Therefore, the controller design is more important for quadruped robots. A robust controller is necessary for the stability analysis and trajectory planning of the system for quadruped robots.

There are a lot of current studies about quadruped robots. For example; BigDog [1] is developed by Boston Dynamics, ANYmal [2] is developed by Hutter et al., Jinpong [3] is developed by Cho et al., HyQ2Max [4] is developed by Semini et al.

The PID controller operates the large share of the control system in the world. Tuning of PID gains using a search algorithm is ensure better response more than traditional methods such as trial-error methods and Ziegler-Nichols method. Detail studies about the optimization of PID controllers are available in the literature. Das et.al. [5] designed a PID controller for an adopted second order DC motor system and they used the evolutionary algorithm based on GWO algorithm to optimize the PID controller. Madadi and Motlagh [6], GWO Algorithm is utilized and designed for DC Motor drive to discover the global optimum solution in search space. Kanojiya and Meshram [7], present a method to determine the optimal PI controller gains on DC motor drive system using PSO algorithm.

Moura Oliveira [8], The GWO algorithm is proposed a PID controllers design using two degrees of freedom control configuration. Verma et.al. [9], presents a novel evolutionary technique which is the GWO algorithm to optimize the parameters of fractional order controller. Lal et.al. [10], present a meta-heuristic optimization algorithm has been applied to interconnected Hydro-thermal power system for automatic generation control. The optimal gains of the fuzzy based PID controllers are obtained by employing the proposed GWO algorithm. Tsai et.al. [11], proposed a novel multi-objective method for optimal robot path planning which based on GWO algorithm. Razmjooy et. al. [12] tuned a LQR controller using GWO algorithm for a single-link flexible joint robot manipulator. Hultmann and do Santos [13], developed a multiobjective non-dominated sorting GA for the tuning of a PID controller applied to a robotic manipulator. Krohling and Rey [14] present a PID controller based on GA for solving the constrained optimization problem in a servo motor system.

This paper examines evaluates the performance of an optimized two PID controllers using GWO algorithm, PSO algorithm and GA for single footstep controlling the quadruped robot in a desired trajectory. The quadruped robot is built SolidWorks and exported to MATLAB/SimMechanics environment. The control system is modelled MATLAB/Simulink environment and PID controllers are tuned with proposed algorithms. The main aim is to use a search algorithm include GWO algorithm, 
PSO algorithm and GA to design a suitable PID controllers for the quadruped robot system. Also, a comparison between the tuning techniques presents to show the best method.

Followed by introduction the paper is created as follows: the model of the quadruped robot is explained in Section 2. Section 3, describes GWO algorithm, PSO algorithm and GA. In section 4, designing the PID controllers optimally for the quadruped robot is shown; applying of the described algorithms is also presented in this section. Simulation results and comparisons are presented in section 5. Finally, in section 6, the paper is concluded.

\section{MODELLING OF QUADRUPED ROBOT}

This chapter presents the specifications of the quadruped robot. The computer aided design (CAD) model of the quadruped robot system built in SolidWorks software. The model which mainly based on the inspiration by nature and other robots shows in Figure 1. The robot has four legs with three DOF of each leg. This structure is simple and allows the robot to perform a wide range of tasks. The physical parameters of the system are given in Table 1. Physical specifications describe the robot's size and weight.

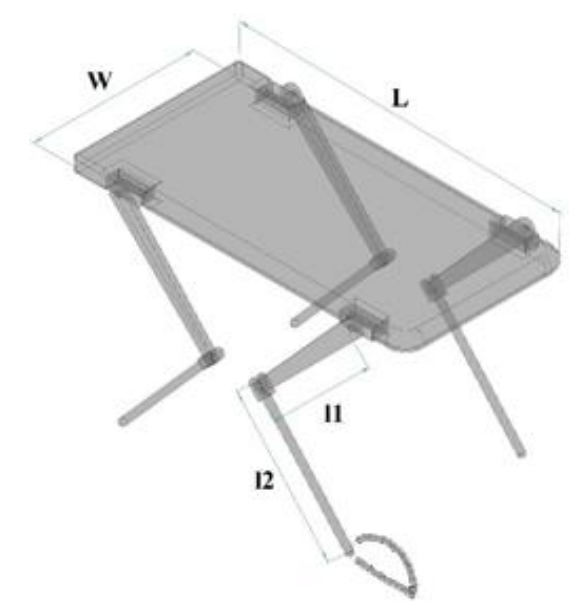

Figure 1: The CAD model of system

TABLE I

THE PHYSICAL PARAMETERS OF SYSTEM

\begin{tabular}{|c|c|}
\hline Parameter & Value \\
\hline Material & Alloy 1060 \\
\hline Weight & $28.7 \mathrm{~kg}$ \\
\hline $\boldsymbol{W}$ & $500 \mathrm{~mm}$ \\
\hline $\boldsymbol{L}$ & $1000 \mathrm{~mm}$ \\
\hline $\boldsymbol{l} \boldsymbol{l}$ & $400 \mathrm{~mm}$ \\
\hline $\boldsymbol{2}$ & $410 \mathrm{~mm}$ \\
\hline
\end{tabular}

\section{DESCRIBED PROPOSED ALGORITHMS}

In this section, GWO algorithm, PSO algorithm and GA summarized. In this paper, the proposed algorithms are used to tune of PID controller gains to provide a single footstep control in a desired trajectory for the quadruped robot.

\section{A. Grey Wolf Optimizer (GWO) Algorithm}

GWO algorithm [15 - 19] is a new meta-heuristic algorithm which is introduced in 2014 by Mirjalili et al. The GWO algorithm mimics the leadership hierarchy and hunting mechanism of the grey wolves in the wild. The method simulates the social hierarchy and hunting behaviour in the society of grey wolves. Four types of simulations are applied in grey wolf hierarchy: Alpha $(\alpha)$, Beta $(\beta)$, Delta $(\delta)$ and Omega $(\omega)$ as shown in Figure 2. The Alpha $(\alpha)$ wolf which leaders of whole group is mostly responsible for making decisions about hunting, sleeping place, time to wake etc. Beta $(\beta)$ wolf which subordinate of Alpha $(\alpha)$ wolves is found in the second rank in the hierarchy. Beta ( $\beta$ ) wolf is known as an Alfa $(\alpha)$ assistant in decision to hunting and other activities. The lowest ranking grey wolf is Omega $(\omega)$ wolf which follow Alphas $(\alpha)$, and Betas ( $\beta$ ), but dominate Omegas ( $\omega)$. If a wolf is not an Alpha $(\alpha)$, Beta $(\beta)$, or Omega $(\omega)$, it is called Delta $(\delta)$ wolf. The search in GWO starts with wolves' population (solutions) which are generated randomly. These wolves estimate the location of prey (optimum) through an iterative procedure in during the hunting (optimization) process. Alpha $(\alpha)$ is the fittest solution followed by Beta $(\beta)$ and Delta $(\delta)$ as the second and third best solutions. The rest of the solutions are least important and considered as Omega $(\omega)$ [15].

The hunting behavior is mainly divided into three steps [15]:

a) Tracking, chasing and approaching the prey.

b) Encircling and harassing the prey until it stops moving.

c) Attacking the prey.

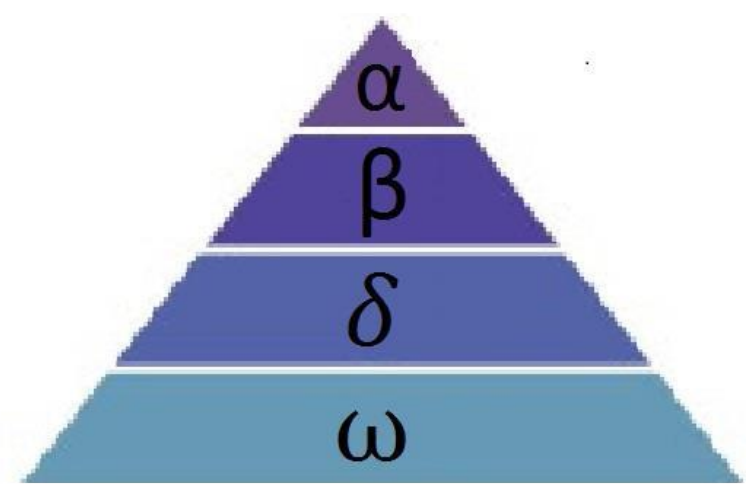

Figure 2: The grey wolf hierarchy [15]

The following equations are proposed in order to mathematically model the encircling behaviour:

$\vec{D}=\left|\vec{C} \overrightarrow{X_{P}}(t)-\vec{X}(t)\right|$

$\vec{X}(t+1)=\overrightarrow{X_{P}}(t)-\vec{A} \vec{D}$

where $t$ is the current iteration, $\vec{A}$ and $\vec{C}$ are coefficient vectors, $\overrightarrow{X_{P}}(t)$ represents the position vector of the victim. $\vec{X}$ indicates the position vector of a grey wolf. The vectors $\vec{A}$ and $\vec{C}$ calculated as follows: 
$\vec{A}=2 \vec{a} \vec{r}_{1}-\vec{a}$

$\vec{C}=2 \vec{r}_{2}$

where $\vec{a}$ include are linearly decreased from 2 to 0 over the course of iterations and $\vec{r}_{1}$ and $\vec{r}_{2}$ are random vectors in the range $[0,1]$.

In GWO, the first three obtained best solutions are saved so far and compel the other search agents (including the omegas) to update their positions due to the position of the best search agents. For this regard, the following formulas are proposed.

$\vec{D}_{\alpha}=\left|\vec{C}_{1} \overrightarrow{X_{\alpha}}-\vec{X}\right|, \vec{D}_{\beta}=\left|\overrightarrow{C_{2}} \overrightarrow{X_{\beta}}-\vec{X}\right|, \vec{D}_{\delta}=\left|\vec{C}_{3} \overrightarrow{X_{\delta}}-\vec{X}\right|$

$\vec{X}_{1}=\overrightarrow{X_{\alpha}}-\vec{A}_{1}\left(\vec{D}_{\alpha}\right), \vec{X}_{2}=\overrightarrow{X_{\beta}}-\vec{A}_{2}\left(\vec{D}_{\beta}\right), \vec{X}_{3}=\overrightarrow{X_{\delta}}-\vec{A}_{3}\left(\vec{D}_{\delta}\right)$

$\vec{X}(t+1)=\frac{\vec{X}_{1}+\vec{X}_{2}+\vec{X}_{3}}{3}$

Pseudo code of the GWO is shown the Figure 3. In GWO algorithm, the mathematical models of the social hierarchy consist of; tracking, encircling, and attacking prey are description in Mirjalili et al. study [15]. The parameters of GWO algorithm are given in Table 2.

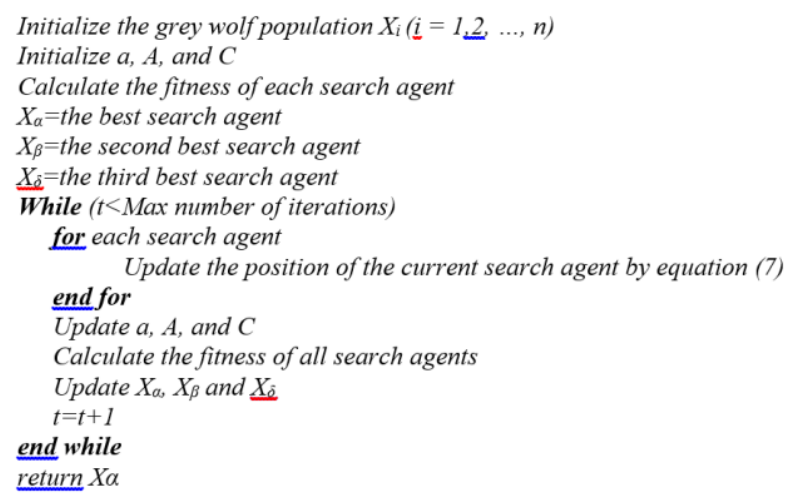

Figure 3: Pseudo code of the GWO algorithm [15]

TABLE II

GREY WOLF OPTIMIZER PARAMETERS

\begin{tabular}{|l|c|}
\hline Maximum Iteration & 100 \\
\hline Number of Search Agent & 30 \\
\hline
\end{tabular}

\section{B. Partcle Swarm Optimization (PSO)}

PSO algorithm [20] which is inspired by social behaviour of bird flocking or fish schooling is one of the most popular optimization algorithms which have been developed in 1995 by Kennedy and Eberhart. In this approach optimal solution to a mathematical optimization problem is restricted of birds behave in the moment the food pursue, the escape from hunters and the search for mates. In the last years, PSO algorithm has been employed in wide variety of applications ranging from classical mathematical programming problems to scientific optimization problems and highly proprietary engineering [21, 22].
Traditional PSO algorithm starts with an initial population (swarm) of candidate solution (particles). The particles look for throughout the search space because of defined formulations. In the wake of seeking, the particles move to their own best known position in the search space and the swarm's best known position. The particles will the guide the other particles movements after finding the best position. Until the satisfactory solution will finally be detected, the searching about the search space is repeated. In each iteration, the swarm is tuned in order to the following equations:

$$
\begin{aligned}
& v_{i}^{t+1}=\omega v_{i}^{t}+c_{1} r_{1}\left(p_{i}^{t}-x_{i}^{t}\right)+c_{2} r_{2}\left(g_{i}^{t}-x_{i}^{t}\right) \\
& x_{i}^{t+1}=x_{i}^{t}+v_{i}^{t+1} \quad \mathrm{i}=1,2, \ldots, \mathrm{n}
\end{aligned}
$$

Where, $t$ is the iteration number, $n$ is the number of particles, , $C_{1}$ and $C_{2}$ are the positive constants, $w$ is the weighted inertia, $r_{1}$ and $r_{2}$ are two random numbers distributed within the range $[0,1], p_{i}$ is the best position of the $i t h$ particle and $g_{i}$ is the best particle among the group members. The parameters of PSO algorithm are given in Table 3 .

TABLE III

\begin{tabular}{|c|c|c|}
\hline \multicolumn{2}{|c|}{ Number of Particle (NoP) } & 30 \\
\hline \multicolumn{2}{|c|}{ Maximum Iteration } & 100 \\
\hline \multicolumn{2}{|c|}{ Velocity Clamping Parameter $\left(V_{\max }\right)$} & 6 \\
\hline \multirow{2}{*}{ Inertia Weight } & $w_{\text {init }}$ & 0.2 \\
\hline & $\boldsymbol{w}_{\text {final }}$ & 0.9 \\
\hline \multicolumn{2}{|c|}{ The Importance of Personal Best Value $\left(c_{1}\right)$} & 2 \\
\hline \multicolumn{2}{|c|}{ The Importance of Neighbourhood Best Value $\left(c_{2}\right)$} & 2 \\
\hline
\end{tabular}
PARTICLE SWARM OPTIMIZATION PARAMETERS

\section{Genetic Algorithm (GA)}

The Genetic Algorithm [23] is an extensively used optimization method based on the process that mimics natural selection and genetics.

In this study, GA Global Optimization Toolbox which is a one of the built-in method in MATLAB Global Optimization Toolbox [24] is used. MATLAB Global Optimization Toolbox is a powerful tool to solve optimization problems. The parameters of GA are given in Table 4.

TABLE IV

\section{GENETİC ALGORITHM PARAMETERS}

\begin{tabular}{|l|c|}
\hline Population Size & 30 \\
\hline Elite Count & 5 \\
\hline Generations & 100 \\
\hline Crossover Fraction & 0.8 \\
\hline
\end{tabular}




\section{DESIGNING AND OPTIMIZATION OF THE PID CONTROLLERS}

In this section, the description of the designing of the PID controllers of the quadruped robot system using GWO algorithm, PSO algorithm and GA is given. Initially, the computer aided design (CAD) of the system are built using SolidWorks and exported to MATLAB/SimMechanics software. The MATLAB/ SimMechanics model of the system is given in Figure 4. The PID controllers are designed in MATLAB/Simulink. Inverse kinematic solutions are used to obtain upper (hip) and lower (knee) leg angular positions from trajectory coordinates. The PID controller model of the quadruped robot is given in Figure 5.

The aim is to search for the optimal values of the gains of PID controllers with respect to a determined objective function which consist of trajectory coordinates. The objective function (J) is:

$J=n o r m \sqrt{|X r e f-X|^{2}+\mid \text { Yref }-\left.Y\right|^{2}}$ where $X_{r e f}$ and $Y_{r e f}$ are the reference coordinate of the trajectory, $X$ and $Y$ are the realized trajectory in simulations. GWO algorithm, PSO algorithm and GA are employed for optimizing the proposed fitness function. The optimisation ranges of gains are set as shown in Table 5.

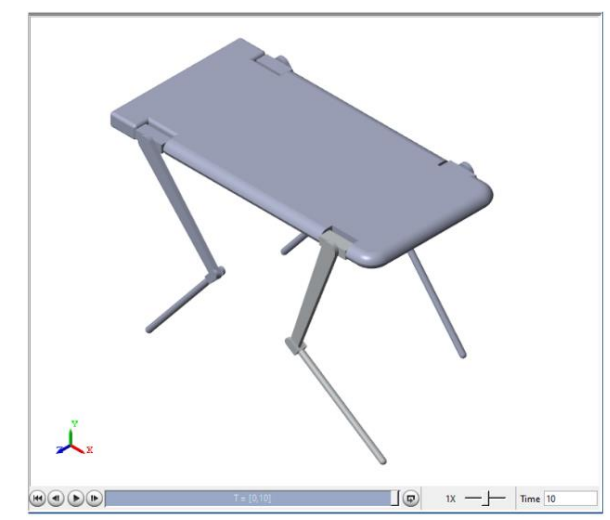

Figure 4: The MATLAB/SimMechanics model of system

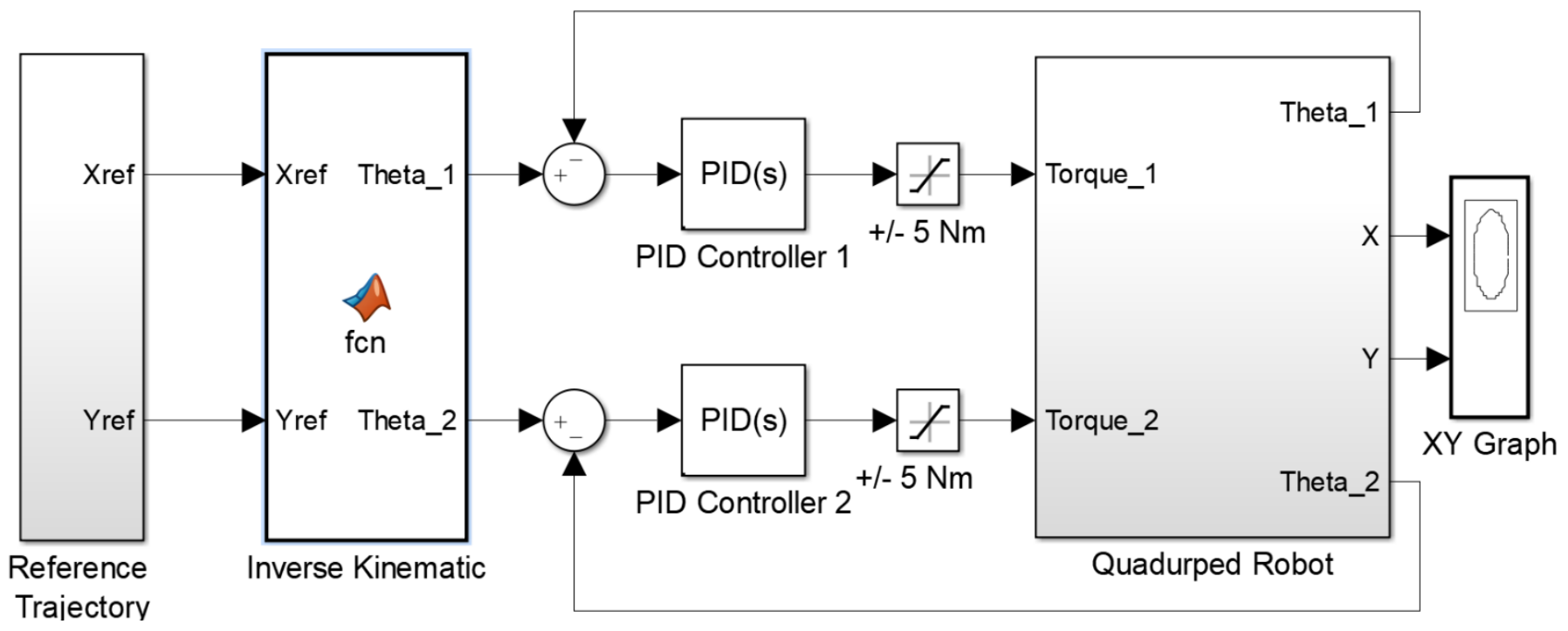

Figure 5: The PID controller model of system

TABLE V

THE RANGE OF PID CONTROLLER'S GAINS

\begin{tabular}{|c|c|c|c|c|c|c|}
\hline & \multicolumn{3}{|c|}{ PID 1 } & \multicolumn{3}{c|}{ PID 2 } \\
\cline { 2 - 7 } & Kp & Ki & Kd & Kp & Ki & Kd \\
\hline Min. & 0 & 0 & 0 & 0 & 0 & 0 \\
\hline Max. & 1000 & 1000 & 1000 & 1000 & 1000 & 1000 \\
\hline
\end{tabular}

\section{SIMULATION RESULTS}

GWO algorithm and PSO algorithm are programmed in MATLAB environment and GA which is a tool in MATLAB Global Optimization Toolbox run on an Intel(R) Core(TM) i74700HQ CPU $2.40 \mathrm{GHz}$ PC with 16.0 GB memory separately. The proposed algorithms run offline for 100 iterations to find the minimum value of the cost function that defined as the objective function $(\mathrm{J})$. After optimization, the best set of the
PID controller's gains values corresponding to the minimum objective function value provided by the optimal GWO algorithm, PSO algorithm and GA are given in Table 6. Also, the minimum objective function value (Best Value) and elapsed time in optimizations are shown in Table 6. The system simulated with tuned gains of the PID controls and the snapshots from MATLAB/SimMechanics simulations are demonstrated in Figure 6. In simulations, upper (hip) and lower (knee) leg angular positions (Theta 1 and Theta 2) are given in Figure 7 and Figure 8. It is clear from the graphics, the proposed algorithms realized in a similar way.

Tracing trajectory performances of the proposed algorithms simulated in MATLAB/Simulink environment. As seen in Figure 9, PID controllers which tuned with GWO algorithm, PSO algorithm and GA ensure settling on the path very successfully. The performances of the proposed algorithms are 
similar. However, as seen in Figure 10, the GWO algorithm traced trajectory with less errors than the others. Objective function convergence performances of the GWO algorithm, PSO algorithm and GA are graphically illustrated in Figure 11. As seen obviously, GWO algorithm is converged better at the end. Moreover, PSO algorithm and GA are converged slower than GWO algorithm. In other terms, GWO algorithm is given better solutions at finding global search and local search according to PSO algorithm and GA.

TABLE VI

THE TUNED GAINS OF PID CONTROLLERS

\begin{tabular}{|c|c|c|c|c|c|c|c|c|}
\hline & \multicolumn{3}{|c|}{ PID 1 } & \multicolumn{3}{c|}{ PID 2 } & \multicolumn{2}{c|}{$\begin{array}{c}\text { Best } \\
\text { Value(J) }\end{array}$} \\
\cline { 2 - 8 } & Kp & Ki & Kd & Kp & Ki & Kd & & \\
\hline GWO & 493.3 & 0.0556 & 8.05 & 93.73 & 21.92 & 3.91 & 63.94 & 1854,59 \\
\hline PSO & 932.50 & 615.00 & 34.85 & 117.40 & 271.36 & 173.95 & 67.93 & 2025.94 \\
\hline GA & 81.65 & 2.54 & 465.37 & 47.78 & 595.40 & 247.91 & 74.14 & 2342.48 \\
\hline
\end{tabular}

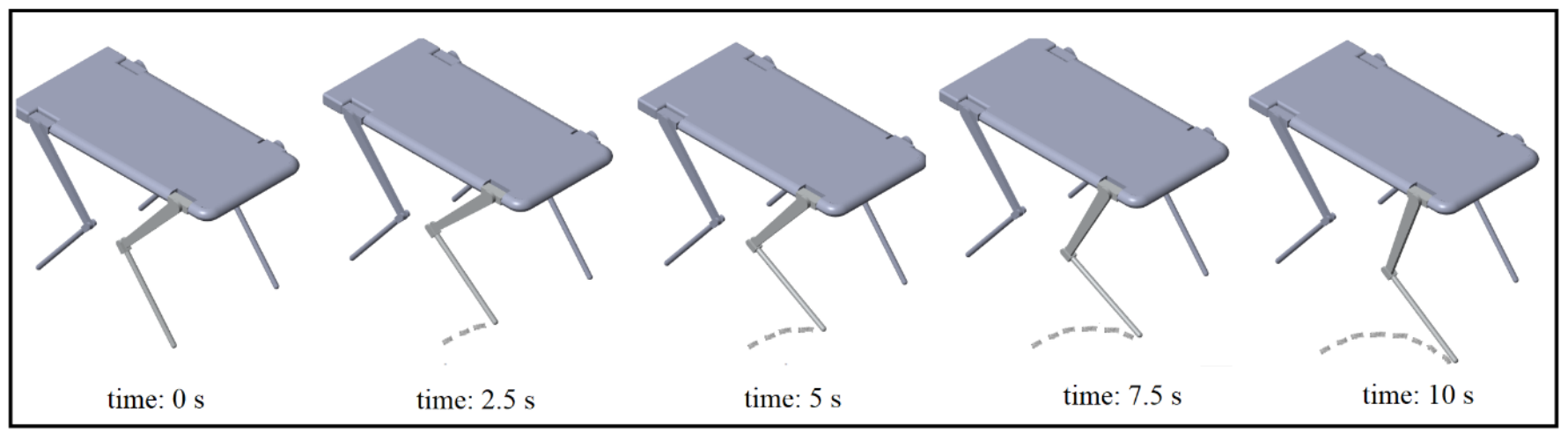

Figure 6: Snapshots from the simulations

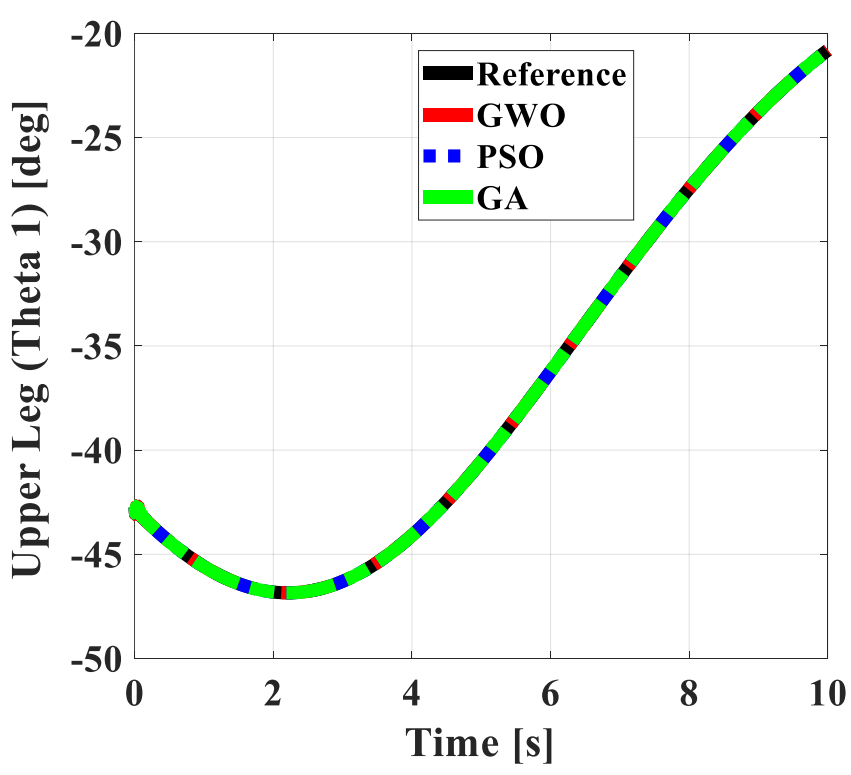

Figure 7: Upper leg angular position of quadruped robot

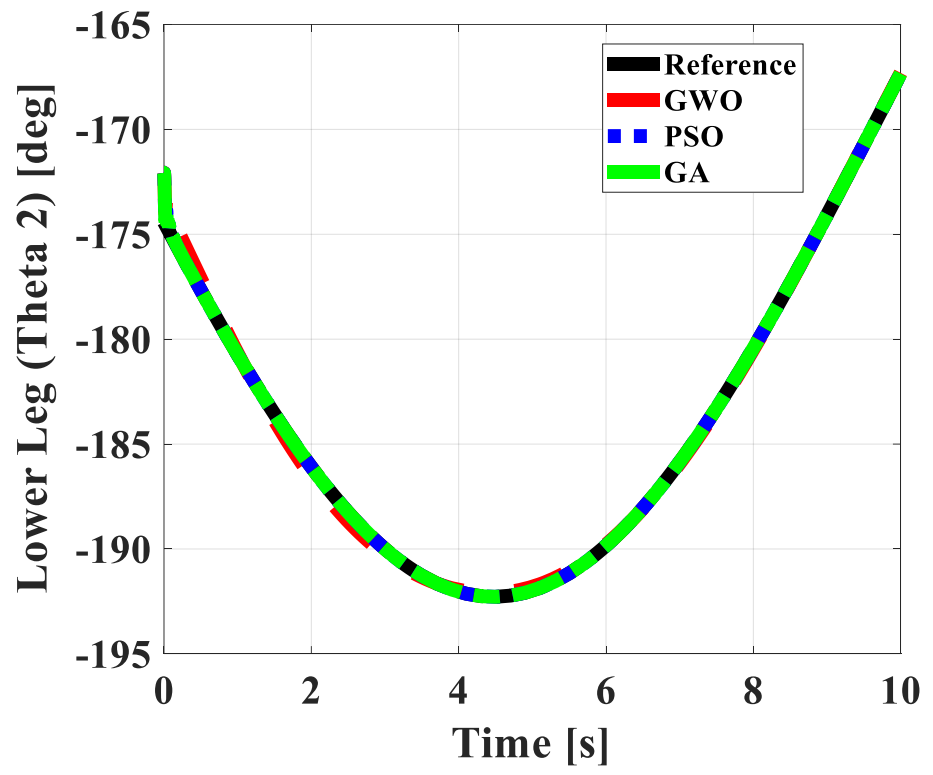

Figure 8: Lower leg angular position of quadruped robot 


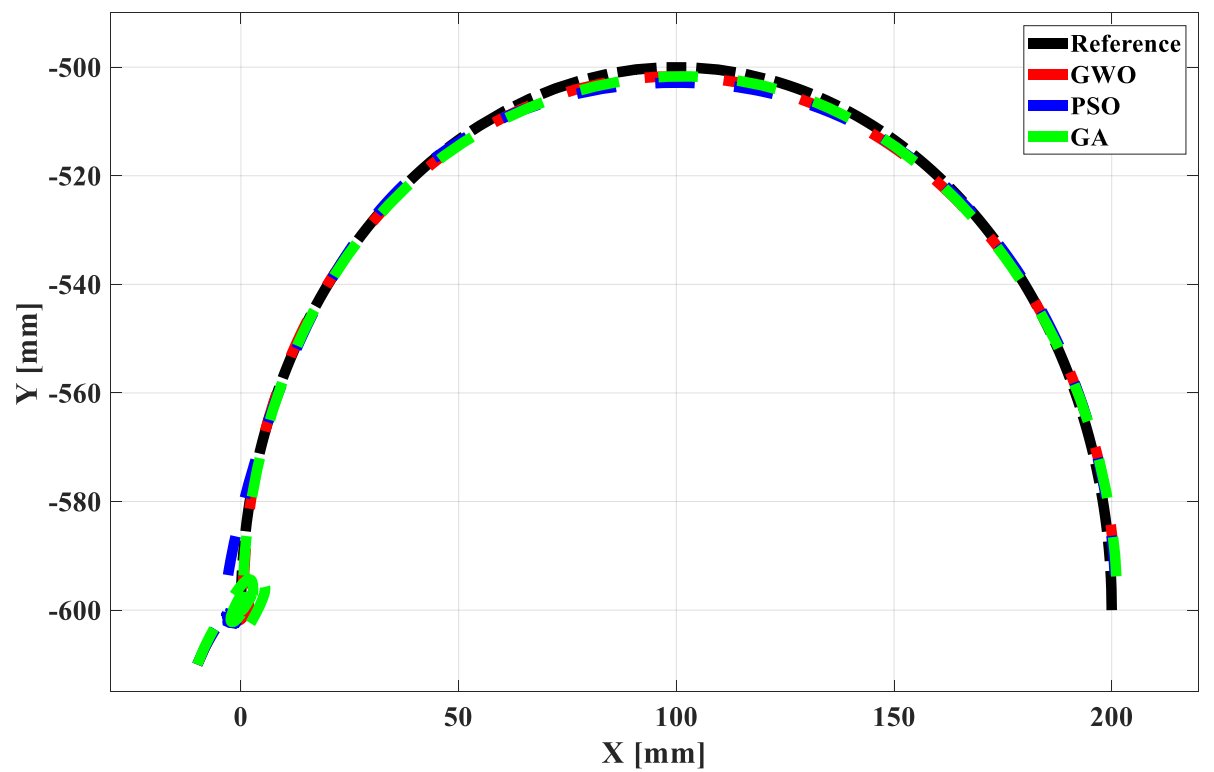

Figure 9: The control performance of the proposed algorithms in trajectory

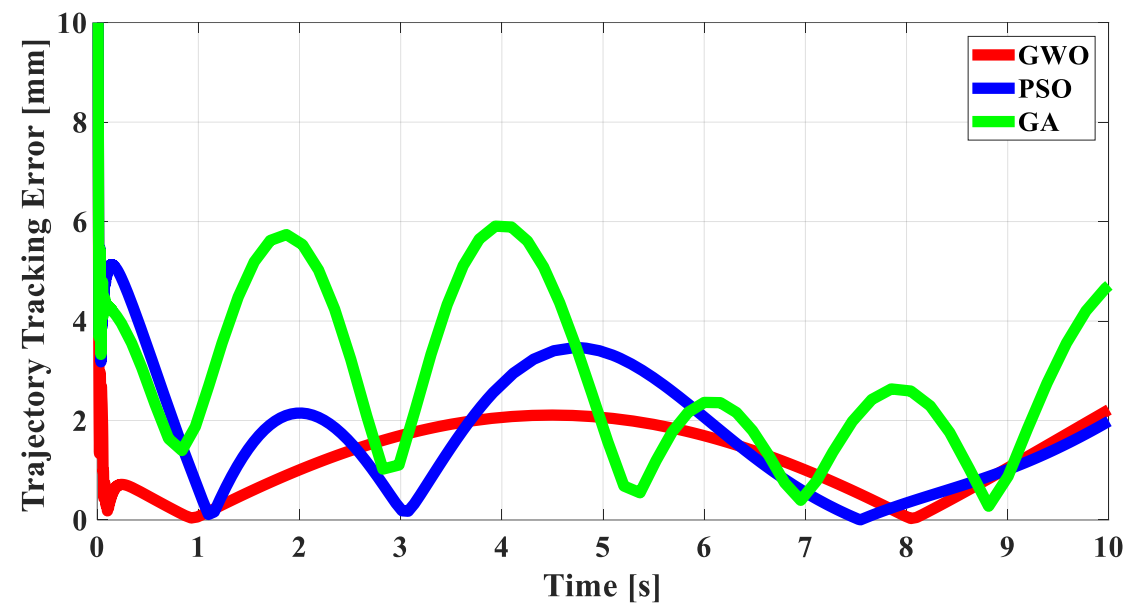

Figure 10: The trajectory tracking error of proposed algorithms

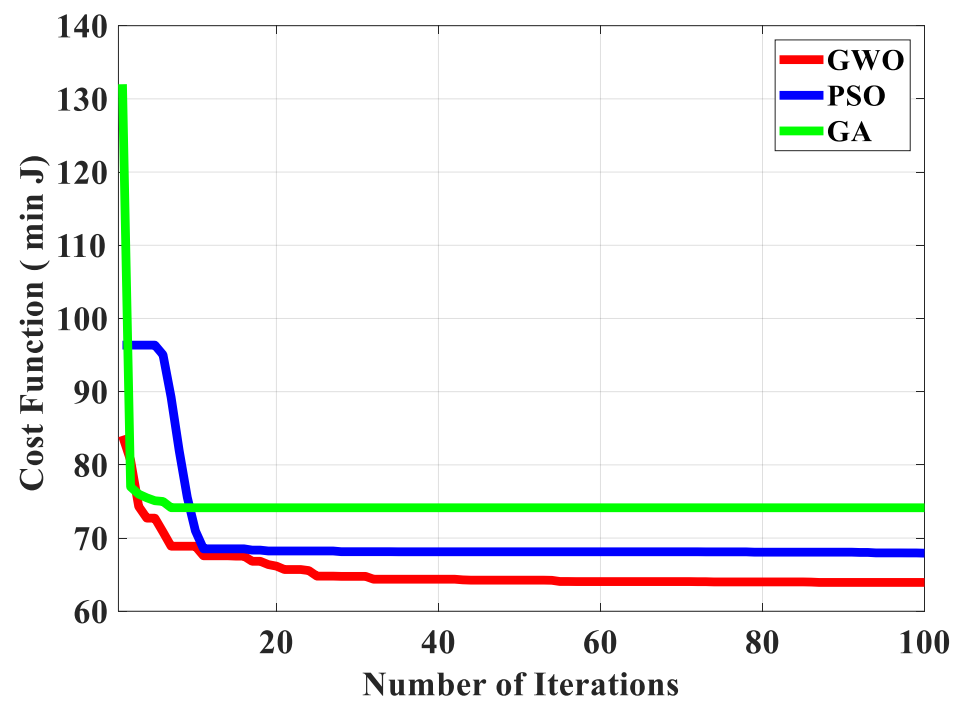

Figure 11: The performance analysis of proposed algorithms 


\section{CONCLUSIONS}

In this paper, the optimization of the PID controllers for the quadruped robot to ensure a single footstep control in a desired trajectory using GWO algorithm, PSO algorithm and GA are investigated by simulations. The importance of this work is that GWO is used first time as a diversity method for a quadruped robot to tune PID controllers. Moreover, to investigate the performance of GWO, it is compared with widespread search algorithms. The GWO algorithm has been successfully applied to design of the PID controllers. In order to demonstrate the performances of GWO algorithm, it is compared with commonly used search algorithms; PSO algorithm and GA. The proposed algorithms are used to find the optimal PID controller gains for the optimum controller performance over the quadruped robot. The simulation results show that GWO algorithm is faster and more efficiency than PSO algorithm and GA in global and local search in optimization. In this study, the GWO algorithm is the best which illustrated satisfactory performances toward PSO algorithm and GA. However, the tuned PID controllers with all the proposed algorithms performed trajectory control of the system successfully and their performances realized similar. It is anticipated that this study will contribute to the quadruped robot works about walking and control.

\section{REFERENCES}

[1] M. Raibert et al., Bigdog, "the rough-terrain quadruped robot", Proceedings of the 17th World Congress The International Federation of Automatic Control, pp. 10822-10825, Seoul, Korea, 2008.

[2] M. Hutter et al., "Anymal-a highly mobile and dynamic quadrupedal robot", Intelligent Robots and Systems (IROS), 2016 IEEE/RSJ International Conference on. IEEE, 2016.

[3] J. Cho et al., "Simple Walking Strategies for Hydraulically Driven Quadruped Robot over Uneven Terrain", Journal of Electrical Engineering \& Technology, vol. 11, no. 5, pp. 1921-718, 2016.

[4] C. Semini et al., "Design of the Hydraulically-Actuated TorqueControlled Quadruped Robot HyQ2Max", IEEE/ASME Transactions on Mechatronics, vol. 22, no. 2, pp. 635-646, 2017.

[5] K. R. Das, D. Das, and Das J., "Optimal tuning of PID controller using GWO algorithm for speed control in DC motor", Soft Computing Techniques and Implementations (ICSCTI), 2015 International Conference on. IEEE, 2015.

[6] A. Madadi and M. M. Motlagh, "Optimal control of DC motor using grey wolf optimizer algorithm”, TJEAS Journal-2014-4-04/373-379, vol. 4, no. 4, pp.373-79, 2014.

[7] R. G. Kanojiya and P. M. Meshram, "Optimal tuning of PI controller for speed control of DC motor drive using particle swarm optimization", Advances in Power Conversion and Energy Technologies (APCET), 2012 International Conference on. IEEE, 2012.

[8] P.B. de Moura Oliveira, H. Freire, and E.J. Solteiro Pires, "Grey wolf optimization for PID controller design with prescribed robustness margins", Soft Computing, vol.20, pp.4243-4255, 2016.

[9] S.K. Verma, S. Yadav, and S.K. Nagar, "Optimization of Fractional Order PID Controller Using Grey Wolf Optimizer", Journal of Control Automation and Electrical Systems, vol. 28, pp. 314-322, 2017. https://doi.org/10.1007/s40313-017-0305-3

[10] D. K. Lal, A. K. Barisal, and M. Tripathy, "Grey wolf optimizer algorithm based fuzzy PID controller for AGC of multi-area power system with TCPS”, Procedia Computer Science, vol. 92, pp. 99-105, 2016.

[11] P. W. Tsai, T. T. Nguyen, T. K. Dao, "Robot Path Planning Optimization Based on Multiobjective Grey Wolf Optimizer", In: Pan JS., Lin JW., Wang CH., Jiang X. (eds) Genetic and Evolutionary Computing. ICGEC
2016. Advances in Intelligent Systems and Computing, Springer, Cham, vol 536, pp.166-173, 2017.

[12] N. Razmjooy, M. Ramezani, and A. Namadchian, "A New LQR Optimal Control for a Single-Link Flexible Joint Robot Manipulator Based on Grey Wolf Optimizer", Majlesi Journal of Electrical Engineering vol.10, no. 3, pp.53-60, 2016

[13] A. H. V. Hultmann, C. L. do Santos, "Tuning of PID Controller Based on a Multiobjective Genetic Algorithm Applied to a Robotic Manipulator", Expert Systems with Applications, vol. 39, pp. 8968-8974, 2012.

[14] R. A. Krohling and J. P. Rey, "Design of optimal disturbance rejection PID controllers using genetic algorithms", IEEE Transactions on Evolutionary Computation, vol. 5, no. 1, pp. 78-82, 2001.

[15] S. Mirjalili, S. M. Mirjalili, and A. Lewis, "Grey wolf optimizer", Advances in Engineering Software, vol. 69, pp. 46-6, 2014.

[16] S. Mirjalili et al., "Multi-objective grey wolf optimizer: a novel algorithm for multi-criterion optimization", Expert Systems with Applications vol. 47, pp.106-119, 2016

[17] Mirjalili S., "How effective is the Grey Wolf optimizer in training multilayer perceptrons, Applied Intelligence", vol. 43, no. 1, pp. 150-161, 2015

[18] S. Shahrzad, S. Z. Mirjalili, and S. M. Mirjalili, "Evolutionary population dynamics and grey wolf optimizer", Neural Computing and Applications vol. 26, no. 5, pp. 1257-1263, 2015.

[19] X. Song, et al., "Grey Wolf Optimizer for parameter estimation in surface waves", Soil Dynamics and Earthquake Engineering vol. 75, pp. 147-157, 2015.

[20] J. Kennedy, "Particle swarm optimization, Encyclopedia of machine learning", Springer US, pp.760-766, 2011.

[21] M. Zarringhalami, S. M. Hakimi and M. Javadi, "Optimal Regulation of STATCOM Controllers and PSS Parameters Using Hybrid Particle Swarm Optimization", IEEE conference, 2010.

[22] S. Panda, and N. Padhy, "Comparison of particle swarm optimization and Genetic Algorithm for FACTS-based controller design", International journal of Applied Soft Computing, pp. 1418-1427, 2008.

[23] S. S. Rao, "Engineering Optimization Theory and Practice", 4th Edition, John Wiley \& Sons Inc. 2009.

[24] MATLAB Global Optimization Toolbox User's Guide (Release 2015b), http://www.mathworks.com/help/gads/index.html

\section{BIOGRAPHIES}

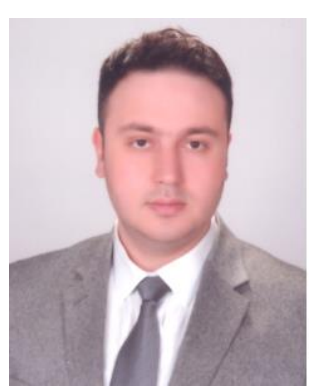

MUHAMMED ARIF ŞEN was born in 1987, Konya, Turkey. He received the B.S. and M.S. degrees in Mechanical Engineering from Selçuk University, Konya, Turkey, in 2010, and 2014, respectively. He currently continues $\mathrm{Ph}$.D. education at Selçuk University Faculty of Engineering, as a research assistant. His research interests include, Control Theory, System Dynamics, Mechatronics Systems, Mobile Robotics, Modelling and Simulation, MATLAB Simulation, Controller Design, The Bees Algorithm and Optimization.

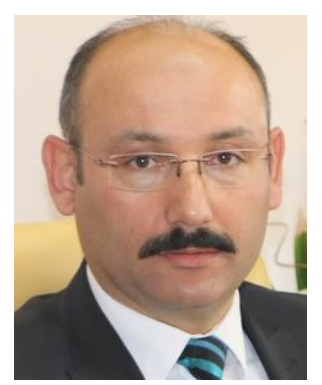

METE KALYONCU received the M.S. and $\mathrm{Ph} . \mathrm{D}$. degrees in Mechanical Engineering from Selçuk University, Konya, Turkey, in 1993 and 1998, respectively. $\mathrm{He}$ is currently a full Professor in the Mechanical Engineering Department, Selçuk University. His research interests include, System Dynamics and Control, Mechatronics, Mechanical Vibration, Robotic, Industrial Design, Fuzzy Logic Control, Modelling of Systems and Simulation, Controller Design, The Bees Algorithm and Optimization. 